\title{
Dark matter and Higgs phenomenology predicted by left-right twin Higgs model in light of CDMS II results
}

\author{
Lei Wang ${ }^{1}$, Jin Min Yang $^{2}$ \\ 1 Department of Physics, Yantai University, Yantai 264005, PR China \\ 2 Key Laboratory of Frontiers in Theoretical Physics, \\ Institute of Theoretical Physics, Academia Sinica, Beijing 100190, PR China
}

\begin{abstract}
The left-right twin Higgs model predicts a light stable scalar $\hat{S}$, which is a candidate for WIMP dark matter. We study its scattering on nucleon and find that the cross section is below the CDMS II upper bound but can reach the SuperCDMS sensitivity. Then we study the Higgs phenomenology by paying special attention to the decay $h \rightarrow \hat{S} \hat{S}$ which is strongly correlated with the dark matter scattering on nucleon. We find that such an invisible decay can be sizable, which can severely suppress the conventional decay modes like $h \rightarrow V V(V=W, Z)$ and $h \rightarrow b \bar{b}$. On the other hand, compared to the SM prediction, the rates of Higgs boson productions at the LHC via gluon-gluon fusion, weak boson fusion or in association with top quark pairs are all reduced significantly, e.g., the gluon-gluon fusion channel can be suppressed by about $30 \%$.
\end{abstract}

PACS numbers: 14.80.Cp,14.80.Ec,12.60.Fr 


\section{INTRODUCTION}

The twin Higgs mechanism [1, 2] is proposed as an interesting solution to the hierarchy problem. The SM Higgs emerges as a pseudo-Goldstone boson once a global symmetry is spontaneously broken, which is similar to what happens in the little Higgs models [3]. An additional discrete symmetry is imposed, which ensures the absence of one-loop quadratic divergence of Higgs mass. The resulting Higgs boson mass is naturally around the electroweak scale when the cut-off scale of the theory is around 5-10 TeV. The twin Higgs mechanism can be implemented in left-right models with the additional discrete symmetry being identified as the left-right symmetry [2]. In the left-right twin Higgs (LRTH) model, several physical Higgs bosons remain after the spontaneous symmetry breaking. Another additional discrete symmetry is introduced in the model under which the $S U(2)_{L}$ doublet $\hat{h}$ is odd while all the other fields are even. The lightest particle $\hat{S}$ in its neutral components is stable and thus can be a candidate for weakly interacting massive particle (WIMP) dark matter. The phenomenology of LRTH model has been studied by some authors [4].

The density of cold dark matter in the universe has been determined precisely by WMAP $[5]$ :

$$
\Omega_{C D M} h^{2}=0.105_{-0.030}^{+0.021}
$$

The thermal production of WIMPs can naturally explain such a relic density. As a direct detection of WIMPs, the CDMS attempts to observe the recoil energy transferred to a target nucleus in an elastic collision with a WIMP. Very recently the CDMS collaboration has completed their analysis of the final data runs of the CDMS II experiment and reported two candidate events [6]. Although these events cannot be interpreted as significant evidence for WIMP interacting with nucleons, the CDMS gives the most stringent upper limit on the WIMP-nucleon spin-independent cross section. For example, the cross section is constrained to be smaller than $3.8 \times 10^{-44} \mathrm{~cm}^{2}$ for a WIMP of $70 \mathrm{GeV}$ at $90 \%$ confidence level [6] . The implications of the new results from the CDMS II experiment have been discussed in many models [7].

In this work we focus on the left-right twin Higgs model. We first examine the scattering of the dark matter candidate $\hat{S}$ with nucleon and compare the rate with the CDMS II results. Then we study the Higgs phenomenology, paying special attention to the decay $h \rightarrow \hat{S} \hat{S}$ which is strongly correlated with the dark matter scattering on nucleon. We will figure 
out the size of such an invisible decay rate and how severely to suppress the conventional decay modes like $h \rightarrow V V(V=W, Z)$ and $h \rightarrow b \bar{b}$. We also study the suppression for the rates of Higgs boson productions at the LHC via gluon-gluon fusion, weak boson fusion or in association with a pair of top quarks. Since the LHC will be able to discover the Higgs boson in the full mass range [8], our study will help to probe the left-right twin Higgs model.

This work is organized as follows. In Sec. II, we briefly review the left-right twin Higgs model. In Sec. III, we examine the scattering of the dark matter candidate $\hat{S}$ with nucleon and compare the rate with the CDMS II results. Also, the correlation of Higgs decays with the dark matter scattering on nucleon is studied. In Sec. IV, we calculate the main productions of the Higgs boson at the LHC. Finally, we give our conclusion in Sec. V.

\section{LEFT-RIGHT TWIN HIGGS MODEL}

\section{A. Mass terms of gauge bosons}

In LRTH model [2, 9], the global symmetry is $U(4) \times U(4)$ with a gauged $S U(2)_{L} \times$ $S U(2)_{R} \times U(1)_{B-L}$ subgroup. The twin symmetry is identified as a left-right symmetry which interchanges $L$ and $R$, implying that that gauge couplings of $S U(2)_{L}$ and $S U(2)_{R}$ are identical $\left(g_{2 L}=g_{2 R}=g_{2}\right)$.

A pair of Higgs fields, $H$ and $\hat{H}$, are introduced and each transforms as $(\mathbf{4}, \mathbf{1})$ and $(\mathbf{1}, \mathbf{4})$ respectively under the global symmetry. They can be written as

$$
H=\left(\begin{array}{c}
H_{L} \\
H_{R}
\end{array}\right), \quad \hat{H}=\left(\begin{array}{c}
\hat{H}_{L} \\
\hat{H}_{R}
\end{array}\right),
$$

where $H_{L, R}$ and $\hat{H}_{L, R}$ are two component objects which are charged under $\mathrm{SU}(2)_{L} \times \mathrm{SU}(2)_{R} \times$ $\mathrm{U}(1)_{B-L}$ as

$$
H_{L} \text { and } \hat{H}_{L}:(\mathbf{2}, \mathbf{1}, 1), \quad H_{R} \text { and } \hat{H}_{R}:(\mathbf{1}, \mathbf{2}, 1)
$$

Each Higgs acquires a non-zero VEV as

$$
<H>=\left(\begin{array}{c}
0 \\
0 \\
0 \\
f
\end{array}\right), \quad<\hat{H}>=\left(\begin{array}{c}
0 \\
0 \\
0 \\
\hat{f}
\end{array}\right)
$$


which breaks one of the $\mathrm{U}(4)$ to $\mathrm{U}(3)$ and yields seven Nambu-Goldstone bosons. The scalar fields can be parameterized as

$$
H=f e^{i \frac{\pi}{f}}\left(\begin{array}{l}
0 \\
0 \\
0 \\
1
\end{array}\right), \quad \text { with } \pi=\left(\begin{array}{cccc}
-N / 2 & 0 & 0 & h_{1} \\
0 & -N / 2 & 0 & h_{2} \\
0 & 0 & -N / 2 & C \\
h_{1}^{*} & h_{2}^{*} & C^{*} & 3 N / 2
\end{array}\right) \text {, }
$$

with $\pi$ being the corresponding Goldstone fields. $N$ is a neutral real pseudoscalar, $C$ and $C^{*}$ are a pair of charged complex scalar fields, and $\left(h_{1}, h_{2}\right)^{T}$ is the SM SU $(2)_{L}$ Higgs doublet. $\hat{H}$ can be parameterized in the same way by its own Goldstone fields $\hat{\pi}$, which contains $\hat{N}$, $\hat{C}$ and $\hat{h}=\left(\hat{h}_{1}^{+}, \hat{h}_{2}^{0}\right)^{T}$.

The generators of $S U(2)_{L} \times S U(2)_{R} \times U(1)_{B-L}$ are given respectively as

$$
\left(\begin{array}{cc}
\frac{1}{2} \sigma_{i} & 0 \\
0 & 0
\end{array}\right), \quad\left(\begin{array}{cc}
0 & 0 \\
0 & \frac{1}{2} \sigma_{i}
\end{array}\right), \quad \frac{1}{2}\left(\begin{array}{cc}
1_{2} & 0 \\
0 & 1_{2}
\end{array}\right),
$$

and the corresponding gauge fields are

$$
W_{2}=\frac{1}{2}\left(\begin{array}{cccc}
W_{L}^{0} & \sqrt{2} W_{L}^{+} & 0 & 0 \\
\sqrt{2} W_{L}^{-} & -W_{L}^{0} & 0 & 0 \\
0 & 0 & W_{R}^{0} & \sqrt{2} W_{R}^{+} \\
0 & 0 & \sqrt{2} W_{R}^{-} & -W_{R}^{0}
\end{array}\right), \quad W_{B-L}=\frac{W_{1}}{2}\left(\begin{array}{cccc}
1 & 0 & 0 & 0 \\
0 & 1 & 0 & 0 \\
0 & 0 & 1 & 0 \\
0 & 0 & 0 & 1
\end{array}\right),
$$

where the Lorentz indices are suppressed. The covariant derivative is

$$
D^{\mu}=\partial^{\mu}-i g_{2} W_{2}^{\mu}-i g_{1} n_{B-L} W_{B-L}^{\mu},
$$

where $g_{1}$ and $g_{2}$ are the gauge couplings for $\mathrm{U}(1)_{B-L}$ and $\mathrm{SU}(2)_{L, R}$, and $n_{B-L}$ is the charge of the field under $\mathrm{U}(1)_{B-L}$.

The covariant kinetic terms of Higgs fields can be written down as [2, 9]

$$
\mathcal{L}_{H}=\left(D_{\mu} H\right)^{\dagger} D^{\mu} H+\left(D_{\mu} \hat{H}\right)^{\dagger} D^{\mu} \hat{H}
$$

with $n_{B-L}=1$. The above Lagrangian contains the following neutral Higgs boson interactions:

$$
\begin{aligned}
\mathcal{L}_{H} & \supset \frac{1}{2} g_{2}^{2} f^{2} s_{1}^{2} W_{L}^{-} W_{L}^{+}+\frac{1}{2} g_{2}^{2}\left(\hat{f}^{2}+f^{2} c_{1}^{2}\right) W_{R}^{-} W_{R}^{+}+\frac{1}{4} g_{1}^{2}\left(f^{2}+\hat{f}^{2}\right) W_{1} W_{1} \\
& -\frac{1}{4} g_{1} g_{2} f^{2}\left(1-c_{2}\right) W_{1} W_{L}^{0}+\frac{1}{8} g_{2}^{2} f^{2}\left(1-c_{2}\right) W_{L}^{0} W_{L}^{0}-\frac{1}{4} g_{1} g_{2}\left(f^{2}+f^{2} c_{2}+2 \hat{f}^{2}\right) W_{1} W_{R}^{0} \\
& +\frac{1}{8} g_{2}^{2}\left(f^{2}+f^{2} c_{2}+2 \hat{f}^{2}\right) W_{R}^{0} W_{R}^{0},
\end{aligned}
$$


where

$$
\begin{aligned}
& c_{1}=\cos \frac{h+v}{\sqrt{2} f}, \quad s_{1}=\sqrt{1-c_{1}^{2}}, \\
& c_{2}=\cos \frac{\sqrt{2}(h+v)}{f}, s_{2}=\sqrt{1-c_{2}^{2}} .
\end{aligned}
$$

The $h$ and $v$ are the SM-like Higgs field and its VEV, respectively, which arise from the $S U(2)_{L}$ doublet $\left(h_{1}, h_{2}\right)^{T}$. For the charged gauge bosons, there is no mixing between $W_{L}^{ \pm}$ and $W_{R}^{ \pm}: W^{ \pm}=W_{L}^{ \pm}$and $W_{H}^{ \pm}=W_{R}^{ \pm}$. At $\mathcal{O}\left(\frac{v^{2}}{f^{2}}\right)$, their masses and Higgs couplings are

$$
\begin{aligned}
m_{W}^{2} & =\frac{1}{4} g_{2}^{2} v^{2}\left(1-\frac{v^{2}}{6 f^{2}}\right), \quad m_{W_{H}}^{2}=\frac{1}{2} g_{2}^{2}\left[\hat{f}^{2}+f^{2}\left(1-\frac{v^{2}}{2 f^{2}}\right)\right], \\
h W W & : \frac{1}{2} g_{2}^{2} v\left(1-\frac{v^{2}}{3 f^{2}}\right), \quad h W_{H} W_{H} \quad: \quad-\frac{1}{2} g_{2}^{2} v\left(1-\frac{v^{2}}{3 f^{2}}\right) .
\end{aligned}
$$

The neutral gauge bosons $Z_{H}, Z$ and $\gamma$ are linear combinations of $W_{L}^{0}, W_{R}^{0}$ and $W_{1}$. Ref. [9] gives the leading-order masses and Higgs couplings for the mass eigenstates. The diagonalization of the gauge mass matrix is performed numerically in our analysis, and the coupling of $h Z Z$ can be obtained at $\mathcal{O}\left(\frac{v^{2}}{f^{2}}\right)$.

\section{B. Mass terms of fermions}

The masses of the first two generation quarks and bottom quark are obtained from the non-renormalizable operators [9]

$$
\mathcal{L}_{Y}=\frac{y_{u}^{\alpha \beta}}{\Lambda}\left(\bar{Q}_{L \alpha} \tau_{2} H_{L}^{*}\right)\left(H_{R}^{T} \tau_{2} Q_{R \beta}\right)+\frac{y_{d}^{\alpha \beta}}{\Lambda}\left(\bar{Q}_{L \alpha} H_{L}\right)\left(H_{R}^{\dagger} Q_{R \beta}\right)+\text { h.c. }
$$

where $\tau_{2}=\left(\begin{array}{cc}0 & -1 \\ 1 & 0\end{array}\right), Q_{L \alpha}=-i\left(u_{L \alpha}, d_{L \alpha}\right)^{T}$ and $Q_{R \alpha}=\left(u_{R \alpha}, d_{R \alpha}\right)^{T}$ with $\alpha$ being the family index. For simplicity, we assume the quark flavor mixing is small and neglect the mixing effects. From Eq. (13), we can get the Higgs boson interactions with the first two generation quarks and bottom quark:

$$
\mathcal{L}_{Y} \simeq-\frac{y_{u}^{\alpha}}{2 \Lambda} f^{2} s_{2} \bar{u}_{L \alpha} u_{R \alpha}-\frac{y_{d}^{\alpha}}{2 \Lambda} f^{2} s_{2} \bar{d}_{L \alpha} d_{R \alpha}+\text { h.c. }
$$

The mass and Higgs coupling of the quark $q$ are given by

$$
m_{q}=\frac{y_{q}}{\sqrt{2}} \frac{f}{\Lambda} v\left(1-\frac{v^{2}}{3 f^{2}}\right), \quad h \bar{q} q:-\frac{m_{q}}{v}\left(1-\frac{2}{3} \frac{v^{2}}{f^{2}}\right)
$$

where $q$ denotes the first two generation quarks or bottom quark. 
For the lepton sector, the Yukawa interaction is similar to Eq. (13), which can generate small masses for the charged leptons and the Dirac mass terms for neutrinos.

For the top quark Yukawa interaction, in order to cancel the one-loop quadratic divergence of Higgs mass induced by the top quark, a pair of vector-like quarks $\left(U_{L}, U_{R}\right)$ are introduced. The Lagrangian can be written as [9]

$$
\mathcal{L}_{t}=y_{L} \bar{Q}_{L 3} \tau_{2} H_{L}^{*} U_{R}+y_{R} \bar{Q}_{R 3} \tau_{2} H_{R}^{*} U_{L}-M \bar{U}_{L} U_{R}+\text { h.c. }
$$

where $Q_{L 3}=-i\left(u_{L 3}, d_{L 3}\right)^{T}$ and $Q_{R 3}=\left(u_{R 3}, d_{R 3}\right)^{T}$. Under left-right symmetry, $y_{L}=y_{R}=y$. From Eq.(16), we can get Higgs interaction as

$$
\mathcal{L}_{t} \simeq-y f s_{1} \bar{u}_{L 3} U_{R}-y f c_{1} \bar{u}_{R 3} U_{L}-M \bar{U}_{L} U_{R}+\text { h.c. }
$$

By diagonalizing the mass matrix in Eq. (17), we obtain the mass eigenstates for the top quark and heavy top quark partner $T$. The field $t_{L}$ and $T_{L}\left(t_{R}\right.$ and $\left.T_{R}\right)$ are the linear combination of $u_{L 3}$ and $U_{L}\left(u_{R 3}\right.$ and $\left.U_{R}\right)$, respectively. The masses and Higgs couplings of the mass eigenstates are given by [9]

$$
\begin{array}{ll}
m_{t}^{2}=\frac{1}{2}\left(M^{2}+y^{2} f^{2}-N_{t}\right), \quad m_{T}^{2}=\frac{1}{2}\left(M^{2}+y^{2} f^{2}+N_{t}\right), \\
h \bar{t} t:-\frac{m_{t}}{v} C_{L} C_{R}, & h \bar{T} T:-\frac{y}{\sqrt{2}}\left(S_{R} S_{L}-C_{L} C_{R} x\right) .
\end{array}
$$

where

$$
\begin{aligned}
S_{L} & =\frac{1}{\sqrt{2}} \sqrt{1-\left(y^{2} f^{2} \cos 2 x+M^{2}\right) / N_{t}}, C_{L}=\sqrt{1-S_{L}^{2}}, \\
S_{R} & =\frac{1}{\sqrt{2}} \sqrt{1-\left(y^{2} f^{2} \cos 2 x-M^{2}\right) / N_{t}}, C_{R}=\sqrt{1-S_{R}^{2}}, \\
N_{t} & =\sqrt{\left(y^{2} f^{2}+M^{2}\right)^{2}-y^{4} f^{4} \sin ^{2} 2 x},
\end{aligned}
$$

with $x=\frac{v}{\sqrt{2} f}$.

\section{Mass term of dark matter}

In addition to the Coleman-Weinberg potential arising from gauge boson contributions, the soft left-right symmetry breaking terms, so called " $\mu$-term", can give masses for $\hat{h}_{1}^{ \pm}$and $\hat{h}_{2}^{0}[9]$ :

$$
V_{\mu}=-\mu_{r}^{2}\left(H_{R}^{\dagger} \hat{H}_{R}+\text { h.c. }\right)+\hat{\mu}^{2} \hat{H}_{L}^{\dagger} \hat{H}_{L} .
$$


In order not to reintroduce fine tuning, $\mu_{r}$ should be less than about $f / 4 \pi$. It is natural for $\hat{\mu}$ not to be much larger than $f$. The masses of $\hat{h}_{2}^{0}$ and $\hat{h}_{1}^{ \pm}$are

$$
\begin{aligned}
M_{\hat{h}_{2}}^{2}= & \frac{3}{16 \pi^{2}}\left[\frac{g_{2}^{2}}{2}\left(\mathcal{Z}\left(M_{W}\right)-\mathcal{Z}\left(M_{W_{H}}\right)\right)+\frac{2 g_{1}^{2}+g_{2}^{2}}{4} \frac{M_{W_{H}}^{2}-M_{W}^{2}}{M_{Z_{H}}^{2}-M_{Z}^{2}}\left(\mathcal{Z}\left(M_{Z}\right)-\mathcal{Z}\left(M_{Z_{H}}\right)\right)\right] \\
& +\mu_{r}^{2} \frac{f}{\hat{f}} \cos x+\hat{\mu}^{2}, \\
M_{\hat{h}_{1}}^{2} \simeq & M_{\hat{h}_{2}}^{2},
\end{aligned}
$$

where $\mathcal{Z}(x)=-x^{2}\left(\ln \frac{\Lambda^{2}}{x^{2}}+1\right)$, and the cut-off scale $\Lambda$ is typically taken to be $4 \pi f$. We neglect the small mass splitting between $\hat{h}_{2}^{0}$ and $\hat{h}_{1}^{ \pm}$due to the electromagnetic interactions. Note that $\hat{\mu}^{2}$ could have either sign, which can allow us to vary the masses of $\hat{h}_{2}^{0}$ and $\hat{h}_{1}^{ \pm}$as a free parameter.

The complex scalar $\hat{h}_{2}^{0}$ can be written as

$$
\hat{h}_{2}^{0}=\frac{\hat{S}+i \hat{A}}{\sqrt{2}},
$$

where $\hat{S}$ and $\hat{A}$ are the scalar and pseudoscalar fields, respectively. We can introduce a new quartic potential term to get the mass splitting between $\hat{S}$ and $\hat{A}$, as well as their Higgs couplings [10]:

$$
V_{H}=-\frac{\lambda_{5}}{2}\left[\left(H_{L}^{\dagger} \hat{H}_{L}\right)^{2}+\text { h.c. }\right] .
$$

From Eq. (23), we can get

$$
\begin{array}{ll}
\delta m_{\hat{S}}^{2}=-\frac{\lambda_{5}}{2} v^{2}\left(1-\frac{v^{2}}{6 f^{2}}\right), & \delta m_{\hat{A}}^{2}=\frac{\lambda_{5}}{2} v^{2}\left(1-\frac{v^{2}}{6 f^{2}}\right), \\
h \hat{S} \hat{S}: \lambda_{5} v\left(1-\frac{v^{2}}{3 f^{2}}\right), & h \hat{A} \hat{A}:-\lambda_{5} v\left(1-\frac{v^{2}}{3 f^{2}}\right) .
\end{array}
$$

Since the quartic terms $-\left(\lambda_{5} / 4\right) h^{2} \hat{S}^{2}$ and $\left(\lambda_{5} / 4\right) h^{2} \hat{A}^{2}$ induced by Eq. (23) have opposite sign, the one-loop quadratic divergence of Higgs mass from the $\hat{S}$ loop and from the $\hat{A}$ loop can be cancelled. Therefore, it is safe to take $\lambda_{5} \sim 1$.

There is also a quartic term which can potentially introduce a mass splitting between $\hat{h}_{2}^{0}$ and $\hat{h}_{1}^{ \pm}[10]$ :

$$
V_{H}^{\prime}=\lambda_{4}\left|\hat{H}_{L}^{\dagger} H_{L}\right|^{2}
$$

However, unlike Eq. (23) if $\lambda_{4}$ is too large, which requires $\left|\lambda_{4}\right| \leq \frac{1}{16 \pi^{2}}$ with $\Lambda=4 \pi f$. Therefore, compared with Eq. 
(24), the corrections of Eq. (25) to the $\hat{h}_{2}^{0}$ mass and Higgs coupling can be neglected. We define two parameters:

$$
\begin{aligned}
\delta_{2} & \equiv m_{\hat{A}}-m_{\hat{S}}=\frac{\lambda_{5}}{\left(m_{\hat{A}}+m_{\hat{S}}\right)} v^{2}\left(1-\frac{v^{2}}{6 f^{2}}\right), \\
\delta_{1} & \equiv m_{\hat{h}_{1}}-m_{\hat{S}}=\frac{\lambda_{5}}{2\left(m_{\hat{h}_{1}}+m_{\hat{S}}\right)} v^{2}\left(1-\frac{v^{2}}{6 f^{2}}\right) .
\end{aligned}
$$

From Eqs. (21), (24) and (26), we can get the relation $\delta_{2} \approx 2 \delta_{1}$ when $m_{\hat{S}}$ is much larger than $\delta_{2}$. Actually, we checked that for a value of $m_{\hat{S}}$ not much larger than $\delta_{2}$, the relation $\delta_{2} \approx 2 \delta_{1}$ is still a good approximation. For example, for $\left(m_{\hat{S}}, \delta_{2}\right)=(34 \mathrm{GeV}, 24 \mathrm{GeV})$, $(70 \mathrm{GeV}, 40 \mathrm{GeV})$ and $(70 \mathrm{GeV}, 20 \mathrm{GeV})$, we found that $\delta_{2} / \delta_{1}$ is $1.77,1.80$ and 1.88 , respectively. In our numerical calculations we assume $\delta_{2}=2 \delta_{1}$ and we checked that the results are changed very little if we take $\delta_{2} / \delta_{1}$ differently as $1.77,1.80$ or 1.88 .

$\hat{S}$ is lighter than $\hat{A}$, and can be a candidate of dark matter. In addition to the Higgs couplings in Eq. (24), the Coleman-Weinberg potential can give the contributions to the couplings of $h \hat{S} \hat{S}, h \hat{A} \hat{A}$ and $h \hat{h}_{1} \hat{h}_{1}$. These expressions are complicated and can be found in [11]. In our analysis, these contributions are considered.

\section{DARK MATTER SCATTERING ON NUCLEON AND HIGGS DECAY}

In LRTH model, the neutral $\hat{S}$ is a candidate for WIMP dark matter. Ref. [10] shows that there are two distinctive mass regions for $\hat{S}$ which can give a relic density in the WMAP $3 \sigma$ range: (i) low mass region, and (ii) high mass region. In this paper we focus on the low mass region where the invisible decay $h \rightarrow \hat{S} \hat{S}$ can be open, which can change other decay branching ratios and thus affect the strategy of searching for the Higgs boson at high energy

colliders. For such a low mass region of $\hat{S}$, Fig. 9 in Ref. [10] shows that in the region satisfying the constraints of $\Gamma_{Z}$ and WMAP $3 \sigma$ relic density, $\delta_{2}$ can vary in the range of 20 $\mathrm{GeV}$ and $40 \mathrm{GeV}$ for $m_{\hat{S}} \approx 70 \mathrm{GeV}$, and for $30 \mathrm{GeV} \lesssim m_{\hat{S}} \lesssim 70 \mathrm{GeV}$ the value of $\delta_{2}$ is around $20 \mathrm{GeV}$.

In LRTH model, the elastic scattering of $\hat{S}$ on a nucleus receives the dominant contributions from the Higgs boson exchange diagrams. The spin-independent cross section between $\hat{S}$ and the nucleon is given by [12]

$$
\sigma_{\hat{S} p(n)}^{S I}=\frac{m_{p(n)}^{2}}{4 \pi\left(m_{\hat{S}}+m_{p(n)}\right)^{2}}\left[f^{p(n)}\right]^{2},
$$


where

with [13]

$$
f^{p(n)}=\sum_{q=u, d, s} f_{T_{q}}^{p(n)} \mathcal{C}_{\hat{S} q} \frac{m_{p(n)}}{m_{q}}+\frac{2}{27} f_{T_{g}}^{p(n)} \sum_{q=c, b, t} \mathcal{C}_{\hat{S} q} \frac{m_{p(n)}}{m_{q}}
$$

$$
\begin{aligned}
& f_{T_{u}}^{(p)} \approx 0.020, \quad f_{T_{d}}^{(p)} \approx 0.026, \quad f_{T_{s}}^{(p)} \approx 0.118, \quad f_{T_{g}}^{(p)} \approx 0.836 \\
& f_{T_{u}}^{(n)} \approx 0.014, \quad f_{T_{d}}^{(n)} \approx 0.036, \quad f_{T_{s}}^{(n)} \approx 0.118, \quad f_{T_{g}}^{(n)} \approx 0.832, \\
& \mathcal{C}_{\hat{S} q}=\frac{g_{h \hat{S} \hat{S}} g_{h \bar{q} q}}{m_{h}^{2}} .
\end{aligned}
$$

Here $g_{h \hat{S} \hat{S}}$ and $g_{h \bar{q} q}$ are the couplings of $h \hat{S} \hat{S}$ and $h \bar{q} q$. Note that $\sigma_{\hat{S} p}^{S I} \approx \sigma_{\hat{S} n}^{S I}$.

In this model the major decay modes of the Higgs boson are the SM-like ones: $h \rightarrow f \bar{f}$ (SM fermion pair), $W W$ and $Z Z$. The LRTH model gives corrections to these decay modes via the corresponding modified Higgs couplings

$$
\Gamma(h \rightarrow X X)=\Gamma(h \rightarrow X X)_{S M}\left(g_{h X X} / g_{h X X}^{S M}\right)^{2}
$$

where $X X$ denotes fermion pairs, $W W$ or $Z Z$. $\Gamma(h \rightarrow X X)_{S M}$ is the decay width in the SM, and $g_{h X X}$ and $g_{h X X}^{S M}$ are the couplings of $h X X$ in the LRTH model and SM, respectively. In our calculations the relevant higher order QCD and electroweak corrections are considered using the code Hdecay [14]. In addition to the SM-like decay modes, the Higgs boson has some new important decay modes which are kinematically allowed in some parameter space: $h \rightarrow \hat{S} \hat{S}, h \rightarrow \hat{A} \hat{A}$ and $h \rightarrow \hat{h}_{1} \hat{h}_{1}$, whose partial widths are given by

$$
\begin{aligned}
\Gamma(h \rightarrow \hat{S} \hat{S}) & =\frac{g_{h \hat{S} \hat{S}}^{2}}{32 \pi m_{h}} \sqrt{1-x_{\hat{S}}}, \\
\Gamma(h \rightarrow \hat{A} \hat{A}) & =\frac{g_{h \hat{A} \hat{A}}^{2}}{32 \pi m_{h}} \sqrt{1-x_{\hat{A}}}, \\
\Gamma\left(h \rightarrow \hat{h}_{1} \hat{h}_{1}\right) & =\frac{g_{h \hat{h}_{1} \hat{h}_{1}}^{2}}{16 \pi m_{h}} \sqrt{1-x_{\hat{h}_{1}}},
\end{aligned}
$$

where $x_{s}=4 m_{s}^{2} / m_{h}^{2}$ with $s=\hat{S}, \hat{A}$ and $\hat{h}_{1}$.

In our calculations, the free parameters involved are $f, \Lambda, M, \mu_{r}, m_{\hat{S}}$ and $\delta_{2}$. $\hat{f}$ can be determined by $f, \Lambda, M$ and $\mu_{r}$ by requiring that the SM Higgs obtains an electroweak symmetry breaking VEV of $246 \mathrm{GeV}$ [9]. The Higgs mass depends on $f, \Lambda, M$ and $\mu_{r}$. Note that in our numerical calculations we used the exact expressions (not only keeping the leading terms of $v^{2} / f^{2}$ ). The Z-pole precision measurements, low energy neutral current process and high energy precision measurements off the Z-pole can give strong constraints 

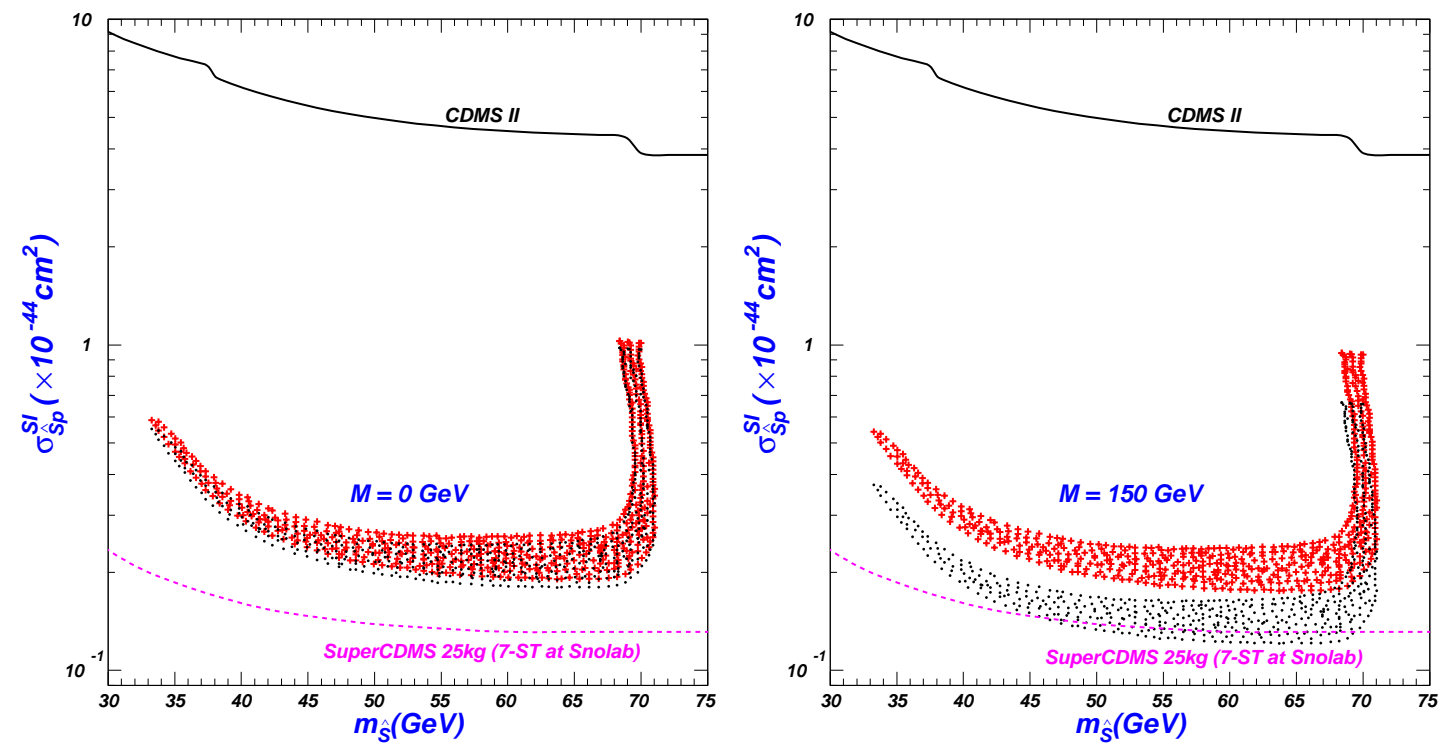

FIG. 1: Scatter plots of $\sigma_{\hat{S} p}^{S I}$ versus $m_{\hat{S}}$ from the scan over the parameters $m_{\hat{S}}$ and $\delta_{2}$ in the region satisfying the constraints of $\Gamma_{Z}$ and WMAP $3 \sigma$ relic density [10]. The lower region denoted by bullets (black) and the upper region denoted by crosses (red) are for $f=500 \mathrm{GeV}$ and $f=1 \mathrm{TeV}$, respectively.
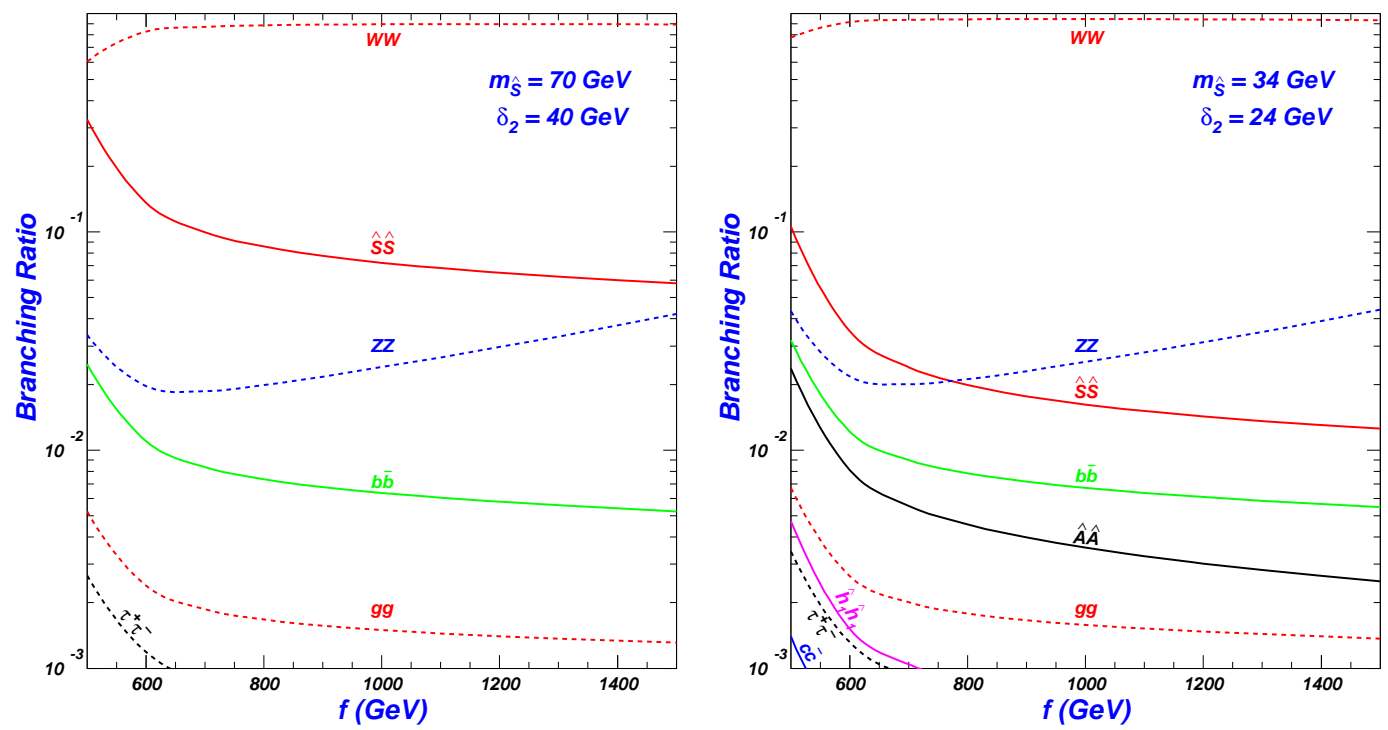

FIG. 2: The Higgs decay branching ratios versus the scale $f$ for $M=0 \mathrm{GeV}$.

on $f$, requiring approximately $f>500 \mathrm{GeV}$. Of course, the fine tuning becomes severe if $f$ is too large [9]. Following Ref. [11], we take the typical parameter space: $500 \mathrm{GeV} \leq f \leq 1500$ $\mathrm{GeV}, \Lambda=4 \pi f, \mu_{r}=50 \mathrm{GeV}$ and $M=0$ (150) GeV, where the Higgs mass is approximately in the range of $160 \mathrm{GeV}$ and $180 \mathrm{GeV}$.

We scan over $m_{\hat{S}}$ and $\delta_{2}$ in the region satisfying the constraints of WMAP $3 \sigma$ relic density 
and $\Gamma_{Z}$. The scatter plots of $\sigma_{\hat{S} p}^{S I}$ versus $m_{\hat{S}}$ are displayed in Fig. 1. We see that $\sigma_{\hat{S} p}^{S I}$ is well below the CDMS II upper bound for the low mass region of $\hat{S}$. The cross section can reach $1.0 \times 10^{-44} \mathrm{~cm}^{2}$ for $m_{\hat{S}} \approx 70 \mathrm{GeV}$ where $\delta_{2}=40 \mathrm{GeV}$ is allowed. The coupling of $h \hat{S} \hat{S}$ increases with $\delta_{2}$, which can enhance $\sigma_{\hat{S} p}^{S I}$ sizably. The parameters $M$ and $f$ can have some effects on $\sigma_{\hat{S} p}^{S I}$ by changing the Higgs mass which can suppress $\sigma_{\hat{S} p}^{S I}$. Besides, the couplings of $h \hat{S} \hat{S}$ and $h q \bar{q}$ increase with $f$ (see Eq. (15) and Eq. (24)), which can give contributions to enhance $\sigma_{\hat{S} p}^{S I}$.

In Fig. 1 we also show the projected sensitivity of SuperCDMS [15]. We see that the LRTH prediction is accessible at SuperCDMS (25kg).

In Fig. 2 we plot the Higgs decay branching ratios versus the scale $f$ for $M=0 \mathrm{GeV}$ (note that the Higgs mass can be determined by the value of $f$, e.g., $m_{h}=159.3 \mathrm{GeV}, 172.6$ $\mathrm{GeV}$ and $178.4 \mathrm{GeV}$ for $f=500 \mathrm{GeV}, 1 \mathrm{TeV}, 1.5 \mathrm{TeV}$, respectively). The left panel shows that $\operatorname{Br}(h \rightarrow \hat{S} \hat{S})$ is subdominant, and the largest value can reach $32 \%$ for $m_{\hat{S}}=70 \mathrm{GeV}$, $\delta_{2}=40 \mathrm{GeV}$ and $f=500 \mathrm{GeV}$. The right panel shows that the new decay modes $h \rightarrow \hat{A} \hat{A}$ and $h \rightarrow \hat{h}_{1} \hat{h}_{1}$ can be open for low values of $m_{\hat{S}}$ and $\delta_{2}$, but their decay branching ratios are relatively small. The branching ratios of all these three new decay modes decrease sizably as $f$ increases. The reason is that the Higgs mass increases with $f$, and the decay width of $h \rightarrow W W$ becomes dominant for the large Higgs mass. The parameter $M$ can have some effects on the Higgs decay modes mainly via changing the Higgs mass, which are not shown here. Besides, $M$ can control the couplings $h t \bar{t}$ and $h T \bar{T}$, which give the dominant contributions to the decay $h \rightarrow g g$. We will show the dependence of the decay $h \rightarrow g g$ on $M$ later.

Fig. 3 shows the scatter plots of $B R(h \rightarrow \hat{S} \hat{S})$ versus $\sigma_{\hat{S} p}^{S I}$ for $M=0 \mathrm{GeV}$ and $M=150$ $\mathrm{GeV}$, respectively. We can see that $B R(h \rightarrow \hat{S} \hat{S})$ is strongly correlated with $\sigma_{\hat{S} p}^{S I}$. When $\sigma_{\hat{S} p}^{S I}$ increases, the corresponding $B R(h \rightarrow \hat{S} \hat{S})$ also becomes large. So the SuperCDMS can probe Higgs decay $h \rightarrow \hat{S} \hat{S}$ via measuring the spin-independent WIMP-nucleon cross section, which is complementary to the exploration of Higgs boson at high energy colliders.

In Fig. 4 we plot $B R(h \rightarrow W W), B R(h \rightarrow Z Z)$ and $B R(h \rightarrow b \bar{b})$ normalized to the SM predictions for several values of $f$. We see that the deviation from the SM prediction for each decay mode is sensitive to $\delta_{2}$, and becomes more sizable as $\delta_{2}$ increases. The corrections to $\operatorname{Br}(h \rightarrow W W)$ and $\operatorname{Br}(h \rightarrow Z Z)$ are almost equal. For $M=0 \mathrm{GeV}, f=500 \mathrm{GeV}, m_{\hat{S}}=70$ $\mathrm{GeV}$ and $\delta_{2}=40 \mathrm{GeV}$, the deviations for the decays $h \rightarrow V V(V=W, Z)$ and $h \rightarrow b \bar{b}$ 

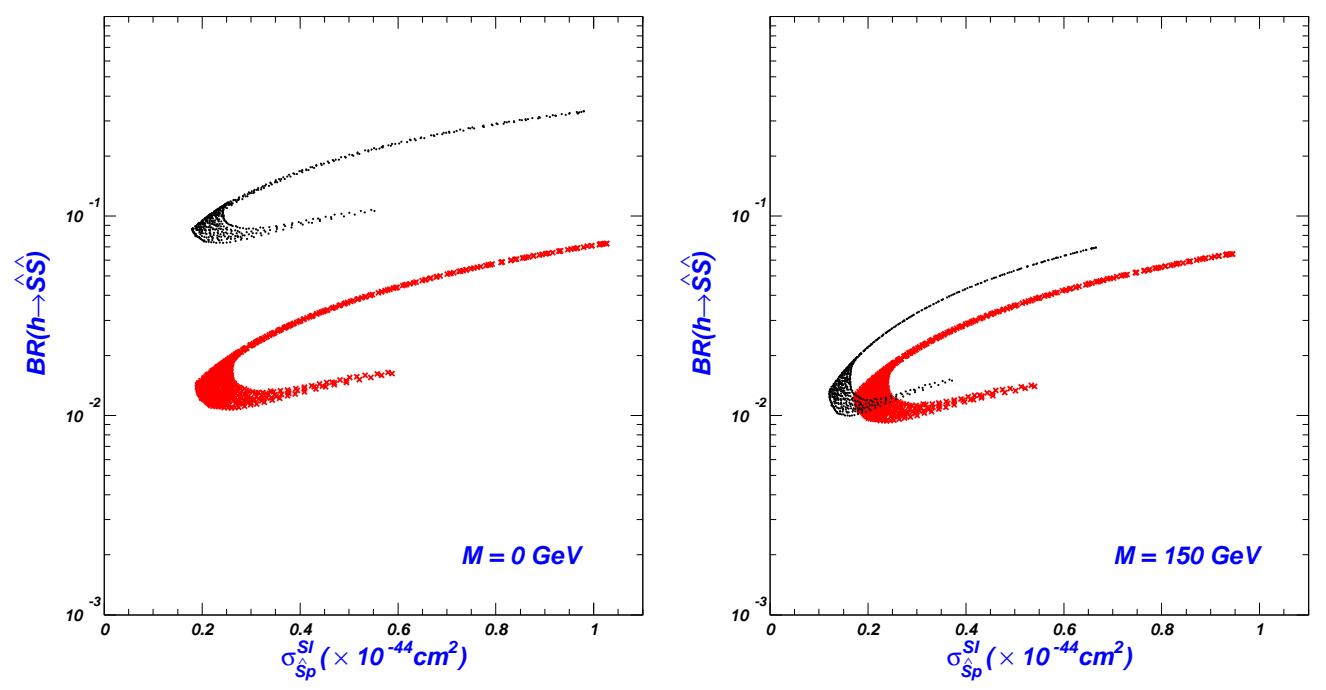

FIG. 3: Same as Fig,1, but projected on the plane of $B R(h \rightarrow \hat{S} \hat{S})$ versus $\sigma_{\hat{S} p}^{S I}$.

can be over $30 \%$ and $47.5 \%$, respectively. The deviations from the SM predictions are also sensitive to $M$ and $f$ which can change Higgs mass.

In LRTH model the Higgs mass is typically in the range of $160-180 \mathrm{GeV}$ and $B R(h \rightarrow \gamma \gamma)$ is severely suppressed. For $\Gamma(h \rightarrow \gamma \gamma)$, the $W$-boson contributions dominate over the top quark contributions [16]. The extra fermions and bosons can give some relatively small corrections, and their contributions tend to cancel each other. Therefore, the modified coupling $h W W$ will give the dominant corrections to $\Gamma(h \rightarrow \gamma \gamma)$, and the suppression of $\operatorname{Br}(h \rightarrow \gamma \gamma)$ approximately equals to that of $\operatorname{Br}(h \rightarrow W W)$, which happens in littlest Higgs model with T-parity [17].

\section{HIGGS PRODUCTION AT LHC}

The Higgs production at the LHC is dominated by the gluon-gluon fusion process. In the SM, the main contributions are from the top quark loop, and the LRTH model can give corrections via the modified coupling of $h \bar{t} t$ and the heavy T-quark loop. The hadronic cross section $\sigma(g g \rightarrow h)$ has a strong correlation with the decay width $\Gamma(h \rightarrow g g)$ :

$$
\begin{aligned}
\sigma(g g \rightarrow h) & =\hat{\sigma}(g g \rightarrow h) \tau_{0} \int_{\tau_{0}}^{1} \frac{d x}{x} f_{g}\left(x, \mu_{F}^{2}\right) f_{g}\left(\frac{\tau_{0}}{x}, \mu_{F}^{2}\right), \\
\hat{\sigma}(g g \rightarrow h) & =\Gamma(h \rightarrow g g) \frac{\pi^{2}}{8 m_{h}^{3}},
\end{aligned}
$$



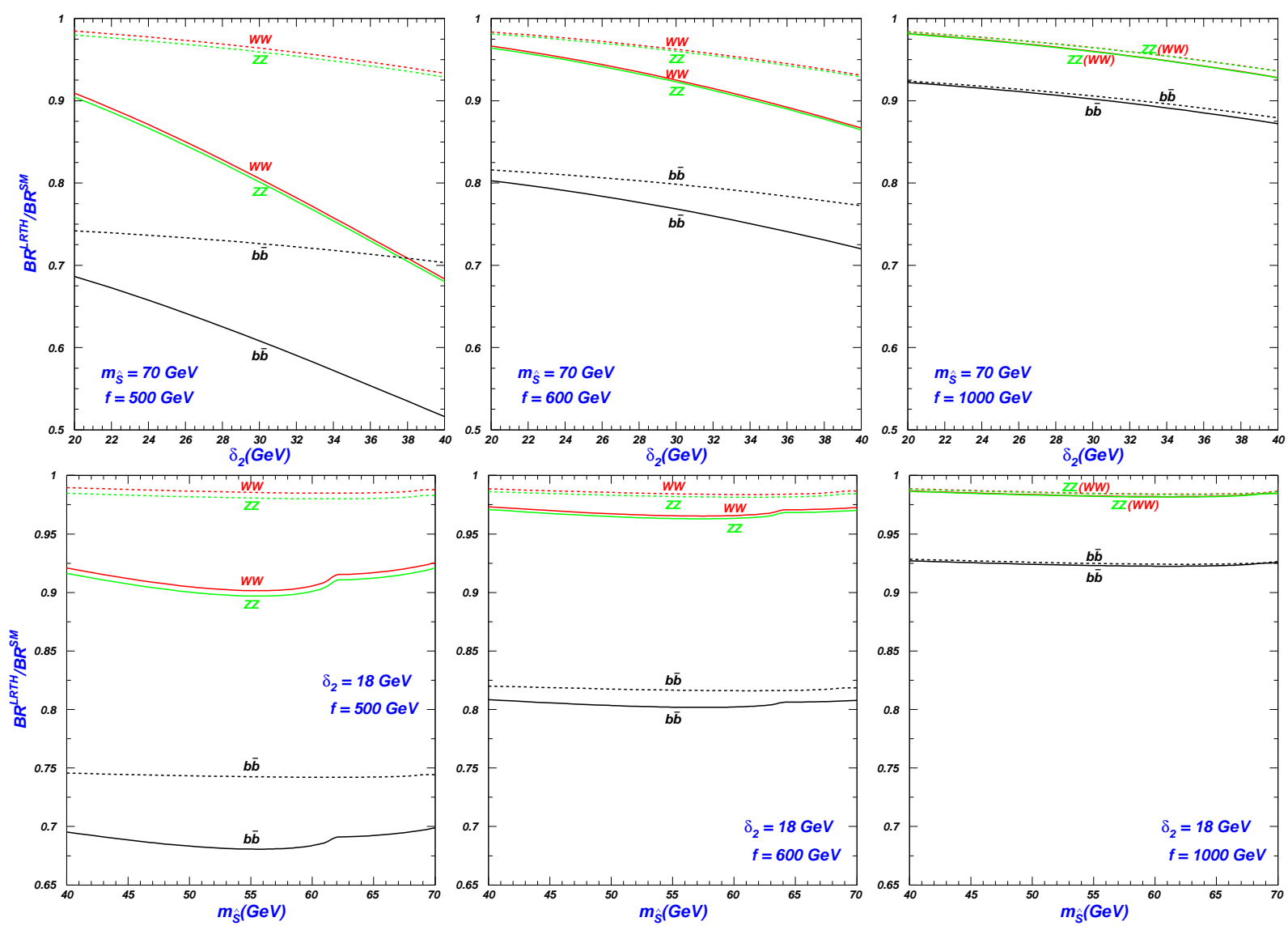

FIG. 4: The decay branching ratios $B R(h \rightarrow W W), B R(h \rightarrow Z Z)$ and $B R(h \rightarrow b \bar{b})$ normalized to the SM predictions. The solid and dashed curves are for $M=0 \mathrm{GeV}$ and $M=150 \mathrm{GeV}$, respectively.

where $\tau_{0}=m_{h}^{2} / s$ with $\sqrt{s}$ being the center-of-mass energy of the LHC. The parton distribution of gluon $f_{g}$ is generated by CTEQ6L [18], and both the renormalization scale $\mu_{R}$ and the factorization scale $\mu_{F}$ are taken as $m_{h}$. From Eq. (32) we get

$$
\frac{\sigma^{L R T H}(g g \rightarrow h)}{\sigma^{S M}(g g \rightarrow h)}=\frac{\Gamma^{L R T H}(h \rightarrow g g)}{\Gamma^{S M}(h \rightarrow g g)} .
$$

The width $\Gamma^{L R T H}(h \rightarrow g g)$ is independent of the parameters $m_{\hat{S}}$ and $\delta_{2}$. In Fig. 5, we plot the ratio $\Gamma^{L R T H}(h \rightarrow g g) / \Gamma^{S M}(h \rightarrow g g)$ versus the scale $f$ for $M=0 \mathrm{GeV}$ and $M=150$ $\mathrm{GeV}$, respectively. We see that, compared with the SM prediction, the LRTH model can suppress the partial width sizably for a small value of $f$. As $f$ gets large, the suppression is weakened. The deviation from the SM prediction is also sensitive to the mixing parameter $M$. For $f=500 \mathrm{GeV}$, the suppression of SM prediction can reach $27 \%$ and $32.5 \%$ for $M=0$ $\mathrm{GeV}$ and $M=150 \mathrm{GeV}$, respectively.

Due to the suppression of the Higgs couplings with gauge bosons and top quark, the 


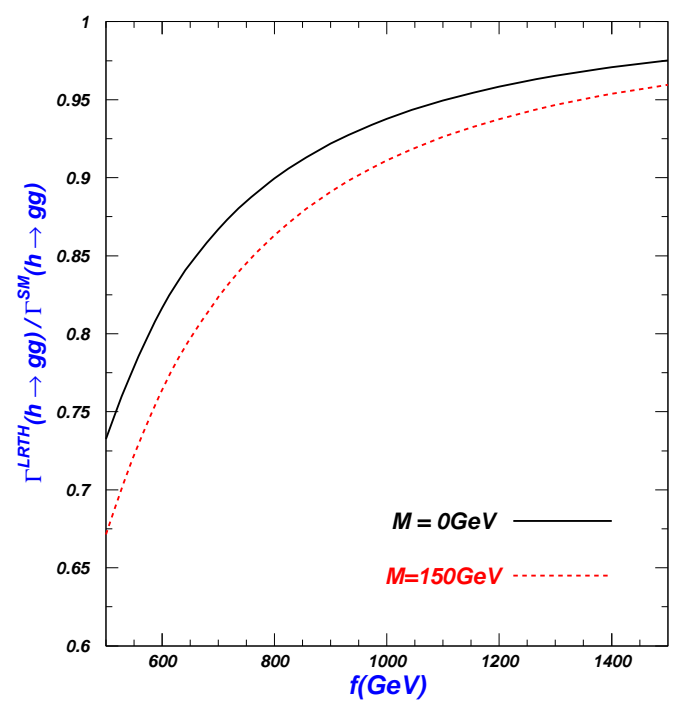

FIG. 5: The ratio $\Gamma^{L R T H}(h \rightarrow g g) / \Gamma^{S M}(h \rightarrow g g)=\sigma^{L R T H}(g g \rightarrow h) / \sigma^{S M}(g g \rightarrow h)$ versus $f$.

Higgs boson production rates via the weak boson fusion or in association with a pair of top quarks are suppressed. Although $\operatorname{Br}(h \rightarrow W W)$ can be sizably suppressed in some parameter space, the decay mode $h \rightarrow W W$ is still an excellent channel for searching for Higgs boson with an intermediate mass [19].

In Table 1 the ratio $\frac{\sigma^{L R T H} \times B R^{L R T H}}{\sigma^{S M} \times B R^{S M}}$ is listed for each main channel: $g g \rightarrow h, V V \rightarrow h$ or $p p \rightarrow h \bar{t} t$ followed by $h \rightarrow V V$ or $h \rightarrow b \bar{b}$. Table 1 shows that such a ratio of events can be sizably suppressed in the LRTH model and the suppression effects could exceed the experimental uncertainty (10\%-20\%) [20]. Therefore, it is possible to probe the LRTH model via these Higgs production modes at the LHC.

Note that similar exotic decays for the SM-like Higgs boson may also be predicted by some other new physics models like the little Higgs models and supersymmetric or two Higgs-doublet models [21]. A common feature of their phenomenology is the suppression of the conventional visible channels of the Higgs boson. To distinguish between different models, all the channels of Higgs production should be jointly analyzed and a linear collider is an ideal machine for such a purpose [22].

Recently both CDF and D0 Collaborations at the Tevatron have searched for the SM Higgs boson and excluded the mass range between $162 \mathrm{GeV}$ and $166 \mathrm{GeV}$ at 95\% CL [23], using all significant production modes: $g g \rightarrow h, V V \rightarrow h, q \bar{q} \rightarrow V h(V=W, Z)$ followed by $h \rightarrow W W$. The productions $g g \rightarrow h$ and $V V \rightarrow h$ are suppressed, similar to the case at the LHC shown in Table 1 . The productions $q \bar{q} \rightarrow V h$ are also suppressed, just like $V V \rightarrow h$, 
TABLE I: The ratio $\frac{\sigma^{L R T H} \times B R^{L R T H}}{\sigma^{S M} \times B R^{S M}}$ for various channels $(g g \rightarrow h, V V \rightarrow h$ or $p p \rightarrow h \bar{t} t$ followed by $h \rightarrow V V$ or $h \rightarrow b \bar{b}$ ) with $m_{\hat{S}}=70 \mathrm{GeV}$ and $\delta_{2}=40 \mathrm{GeV}$.

\begin{tabular}{|c|c|c|c|c|c|c|}
\hline & \multicolumn{3}{|c|}{$h \rightarrow b \bar{b}$} & \multicolumn{3}{c|}{$h \rightarrow V V$} \\
\cline { 2 - 7 } & $f=500 \mathrm{GeV}$ & $f=600 \mathrm{GeV}$ & $f=1 \mathrm{TeV}$ & $f=500 \mathrm{GeV}$ & $f=600 \mathrm{GeV}$ & $f=1 \mathrm{TeV}$ \\
\hline$g g \rightarrow h \quad(M=0 \mathrm{GeV})$ & 0.38 & 0.59 & 0.82 & 0.50 & 0.71 & 0.87 \\
$(M=150 \mathrm{GeV})$ & 0.47 & 0.59 & 0.80 & 0.63 & 0.71 & 0.85 \\
\hline$V V \rightarrow h \quad(M=0 \mathrm{GeV})$ & 0.46 & 0.66 & 0.85 & 0.60 & 0.80 & 0.90 \\
$(M=150 \mathrm{GeV})$ & 0.62 & 0.71 & 0.85 & 0.82 & 0.85 & 0.91 \\
\hline$p p \rightarrow t \bar{t} h(M=0 \mathrm{GeV})$ & 0.50 & 0.70 & 0.87 & 0.66 & 0.85 & 0.92 \\
$(M=150 \mathrm{GeV})$ & 0.59 & 0.69 & 0.85 & 0.79 & 0.83 & 0.90 \\
\hline
\end{tabular}

because these productions are induced by the suppressed couplings $h W W$ and $h Z Z$. Since all these productions $g g \rightarrow h, V V \rightarrow h$ and $q \bar{q} \rightarrow V h$ followed by $h \rightarrow W W$ can be severely suppressed in the LRTH model, the constraints on the Higgs mass from the Tevatron will be largely relaxed in the LRTH model.

\section{CONCLUSION}

In LRTH model, the scalar $\hat{S}$ is a natural candidate for WIMP dark matter, and the Higgs boson mass is typically in the range of $160-180 \mathrm{GeV}$. Since the invisible decay $h \rightarrow \hat{S} \hat{S}$ can affect other decay branching ratios, and also has a strong correlation with the scattering on nucleon, we in this work focused on the low mass region of $\hat{S}$ so that the decay $h \rightarrow \hat{S} \hat{S}$ can be open. We obtained the following observations: (i) The cross section of $\hat{S}$ scattering on nucleon can naturally satisfy the CDMS II upper bound, and can be large enough to be accessible at SuperCDMS; (ii) The Higgs boson can have a sizable invisible decay $h \rightarrow \hat{S} \hat{S}$, whose branching ratio can reach $32 \%$ and has a strong correlation with the cross section of $\hat{S}$ scattering on nucleon. However, the branching ratios of other new decay modes $h \rightarrow \hat{A} \hat{A}$ and $h \rightarrow \hat{h}_{1} \hat{h}_{1}$ are small; (iii) The branching ratios of the conventional decay modes of the Higgs boson, $h \rightarrow V V(V=W, Z)$ and $h \rightarrow b \bar{b}$, can be suppressed over $30 \%$ and $47.5 \%$, respectively; (iV) The Higgs production cross sections times the branching ratios of the 
conventional decays can be all sizably suppressed. So, it is possible to probe the LRTH model via the Higgs productions at the LHC.

\section{Acknowledgment}

We thank Wenyu Wang for discussions and Shufang Su for providing the Calchep Model Code. This work was supported in part by the Foundation of Yantai University under Grant No.WL09B31, by the National Natural Science Foundation of China (NNSFC) under grant Nos. 10821504, 10725526 and 10635030, by the Project of Knowledge Innovation Program (PKIP) of Chinese Academy of Sciences under grant No. KJCX2.YW.W10 and by an invitation fellowship of Tohoku University (Global COE of Japan).

[1] Z. Chacko, H. S. Goh, and R. Harnik, Phys. Rev. Lett. 96, 231802 (2006); R. Barbieri, T. Gregoire, and L. J. Hall, hep-ph/0509242 Z. Chacko, Y. Nomura, M. Papucci, and G. Perez, JHEP 01, 126 (2006); R. Foot and R. R. Volkas, Phys. Lett. B 645, 75 (2007); A. Falkowski, S. Pokorski, and M. Schmaltz, Phys. Rev. D 74, 035003 (2006); S. Chang, L. J. Hall, and N. Weiner, Phys. Rev. D 75, 035009 (2007).

[2] Z. Chacko, H. S. Goh, and R. Harnik, JHEP 01, 108 (2006).

[3] N. Arkani-Hamed, A. G. Cohen and H. Georgi, Phys. Lett. B 513, 232 (2001); N. ArkaniHamed, et al., JHEP 0208, 021 (2002).

[4] See, e.g., D. W. Jung and J. Y. Lee, hep-ph/0701071; Phys. Rev. D 76, 095016 (2007); A. Abada, I. Hidalgo, Phys. Rev. D 77, 113013 (2008); W. Ma, C. X. Yue, and Y. Z. Wang, Phys. Rev. D 79, 095010 (2009); C. X. Yue, H. D. Yang and W. Ma, Nucl. Phys. B 818, 1 (2009); Y. B. Liu, X. L. Wang, Europhys. Lett. 86, 61002 (2009); H. S. Goh, C. A. Krenke, arXiv:0911.5567.

[5] D. N. Spergel et al. [WMAP Collaboration], Astrophys. J. Suppl. 170, 377 (2007).

[6] Z. Ahmed et al. [The CDMS-II Collaboration], arXiv:0912.3592 [astro-ph.CO].

[7] M. Kadastik, K. Kannike, A. Racioppi and M. Raidal, arXiv:0912.3797; N. Bernal and A. Goudelis, arXiv:0912.3905; A. Bottino, F. Donato, N. Fornengo and S. Scopel, arXiv:0912.4025; D. Feldman, Z. Liu and P. Nath, arXiv:0912.4217; M. Ibe and 
T. T. Yanagida, arXiv:0912.4221; R. Allahverdi, B. Dutta and Y. Santoso, arXiv:0912.4329; M. Endo, S. Shirai and K. Yonekura, arXiv:0912.4484; Q. H. Cao, I. Low and G. Shaughnessy, arXiv:0912.4510, Q. H. Cao, C. R. Chen, C. S. Li and H. Zhang, arXiv:0912.4511; K. Cheung and T. C. Yuan, arXiv:0912.4599; J. Hisano, K. Nakayama and M. Yamanaka, arXiv:0912.4701; X. G. He, et al., arXiv:0912.4722; I. Gogoladze, R. Khalid, S. Raza and Q. Shafi, arXiv:0912.5411; M. Aoki, S. Kanemura and O. Seto, arXiv:0912.5536; R. Foot, arXiv:1001.0096; M. Asano and R. Kitano, arXiv:1001.0486; W. S. Cho et al., arXiv:1001.0579; J. Shu, P. F. Yi, S. H. Zhu, arXiv:1001.1076; D. P. Roy, arXiv:1001.4346; S. Khalil, H. S. Lee, E. Ma, arXiv:1002.0692; A. Bandyopadhyay, et al., arXiv:1002.0753, arXiv:1003.0809; J. Hisano, et al., arXiv:1003.3648; Masaki Asano, et al., arXiv:0912.5361.

[8] ATLAS Collaboration, ATLAS Techinical Design Report, CERN/LHC-99-15 (1999).

[9] H. S. Goh, S. Su, Phys. Rev. D 75, 075010 (2007).

[10] E. M. Dolle, S. Su, Phys. Rev. D 77, 075013 (2008).

[11] Avialable at http://www.physics.arizona.edu/ shufang/twinhiggs.html.

[12] R. Barbieri, L. J. Hall and V. S. Rychkov, Phys. Rev. D 74, 015007 (2006); G. Jungman, M. Kamionkowski, and K. Griest, Phys. Rept. 267, 195 (1996); M.A. Shifman, A. I. Vainshtein, and V. I. Zakharov, Phys. Lett. B 78, 443 (1978).

[13] A. Bottino, F. Donato, N. Fornengo, and S. Scopel, Astropart. Phys. 18, 205 (2002); J. R. Ellis, K. A. Olive, Y. Santoso, and V. C. Spanos, Phys. Rev. D 71, 095007 (2005).

[14] A. Djouadj, J. Kalinowski and M. Spira, Computl. Phys. Commun. 108, 56 (2006).

[15] R. Gaitskell, V. Mandic, and J. Filippini, http://dmtools.berkeley.edu/limitplots.

[16] See for instance: M. Spira, Fortsch. Phys. 46, 203 (1998).

[17] R. S. Hundi, B. Mukhopadhyaya, A. Nyffeler, Phys. Lett. B 649, 280 (2007); C. R. Chen, K. Kobe, C. P. Yuan, Phys. Lett. B 640, 263 (2006).

[18] J. Pumplin et al. (CTEQ Collaboration), JHEP 02, 032 (2006).

[19] ATLAS collaboration, ATLAS detector and physics performance, Technical design report. Vol. 2, CERN-LHCC-99-15; S. Asai et al., Eur. Phys. Jour. C 32S2, 19 (2004); D. L. Rainwater and D. Zeppenfeld, Phys. Rev. D 60, 113004 (1999) [Erratum-ibid. D 61, 099901 (2000)].

[20] D. Zeppenfeld, et. al., Phys. Rev. D 62, 013009 (2000); A. Djouadi et al., hep-ph/0002258; A. Belyaev and L. Reina, JHEP 0208, 041 (2002); M. Duehrssen, ATL-PHYS-2003-030; M. Duhrssen, et. al., Phys. Rev. D 70, 113009 (2004). 
[21] For Higgs exotic decays in little Higgs models, see, e.g., K. Cheung, J. Song Phys. Rev. D 76, 035007 (2007); L. Wang and J. M. Yang, Phys. Rev. D 79, 055013 (2009); X.-F. Han, L. Wang, J. M. Yang, Nucl. Phys. B 825, 222 (2010); Phys. Rev. D 78, 075017 (2008). For Higgs exotic decays in SUSY or 2HDM models, see, e.g., R. Dermisek, J. F. Gunion, Phys. Rev. D 75, 075019 (2007). J. Cao, et al., Phys. Rev. D 80, 071701 (2009); Phys. Rev. D 79, 091701 (2009); W. Wang, et al., JHEP 0911, 053 (2009); For Higgs Boson Decay into Massive Neutrinos of 4th Generation, see, e.g., K. Belotsky, et al., Phys. Rev. D 68, 054027 (2003).

[22] For distinguishing little Higgs models via various channels, see, e.g., L. Wang, F. Xu and J. M. Yang, JHEP 1001, 107 (2010). L. Wang and J. M. Yang, Phys. Rev. D 77, 015020 (2008); L. Wang, et al., Phys. Rev. D 76, 017702 (2007); Phys. Rev. D 75, 074006 (2007).

[23] T. Aaltonen, et al. (CDF and D0 Collaborations), Phys. Rev. Lett. 104, 061802 (2010); T. Aaltonen, et al. (CDF Collaboration), Phys. Rev. Lett. 104, 061803 (2010); V. Abazov, et al. (D0 Collaboration), Phys. Rev. Lett. 104, 061804 (2010). 